\title{
Cross antigenicity of immunodominant polypeptides of somatic antigen of Oesophagostomum columbianum with other helminths by western blotting
}

\author{
Sunita Dalal, Arvind Prasad, Abdul Nasir and Vijesh Kumar Saini
}

\author{
Network Program on G.I. Parasitism, Division of Parasitology, Indian Veterinary Research Institute, Izatnagar, Bareilly, \\ Uttar Pradesh, India. \\ Corresponding author: Arvind Prasad, e-mail: drarvindivri@rediffmail.com, SD: drsunita009@gmail.com, \\ AN: drnasirivri@rediffmail.com, VKS: vijesh24@gmail.com \\ Received: 20-06-2015, Revised: 17-09-2015, Accepted: 24-09-2015, Published online: 05-11-2015
}

doi: 10.14202/vetworld.2015.1279-1285 How to citethis article: DalalS, PrasadA, NasirA, Saini VK(2015)Crossantigenicity of immunodominant polypeptides of the somatic antigen of Oesophagostomum columbianum with other helminths by western blotting, Veterinary World 8(11): 1279-1285.

\begin{abstract}
Aim: Oesophagostomum columbianum in small ruminants in India is found as mixed infection commonly in sheep and goat. Haemonchus contortus, an abomasal nematode is found as concurrent infection with it. Eggs of Haemonchus and $O$. columbianum cannot be easily distinguished. Diagnosis of $O$. columbianum may only be possible if a non-cross antigenic polypeptide was available for immunodiagnosis.

Materials and Methods: Somatic antigen (SoAg) of $O$. columbianum was fractionated by sodium dodecyl sulfatepolyacrylamide gel electrophoresis and immunodominant polypeptides were identified by western blotting with homologous hyperimmune serum (HIS) and experimental sera of sheep or goat infected with other helminths.

Results: SoAg of $O$. columbianum was immunoaffinity purified. Sharp polypeptide bands of 130, 72 and 68 KDa were observed along with several faint bands of lower molecular weight. Western blot of purified SoAg of O. columbianum with homologous HIS showed reaction with all the protein bands of 17, 28, 30, 32, 35, 38, 50, 68, 100, 130, 150, and 170 kDa. For identification of non-cross antigenic polypeptide, immunoaffinity purified SoAg of $O$. columbianum was reacted to heterologous HIS against $H$. contortus, Paramphistomum epiclitum, and Fasciola gigantica in western blotting utilizing completely dry method (i-blot). Among high molecular weight polypeptides 100 and $150 \mathrm{kDa}$ were non-cross antigenic and among low molecular weight except $50 \mathrm{kDa}$ polypeptide, $17,30,32,35$, and $38 \mathrm{kDa}$ of $O$. columbianum were not cross antigenic with other helminths.
\end{abstract}

Conclusions: Hence, polypeptides of $17,30,32,35$ and $38 \mathrm{kDa}$ as well as 100 and $150 \mathrm{kDa}$ polypeptides of $O$. columbianum may be exploited for immunodiagnosis of the infection in sheep and goat with extensive studies on cross antigenicity.

Keywords: cross antigenicity, hyperimmune sera, immunodiagnostic polypeptides, Oesophagostomum columbianum.

\section{Introduction}

Oesophagostomum columbianum, commonly known as pimply gut worm causes severe damage to the gut of sheep and goat causing diarrhea. It is one of the most prevalent highly pathogenic and economically important nematode parasite throughout the Asian subcontinent [1]. O. columbianum is more common in tropical and subtropical countries where temperature and humidity are much higher compared to temperate countries. It is also different from other nematodes in the respect that adults are not pathogenic residing in the lumen of the large intestine whereas $\mathrm{L}_{4}$ larvae remain in nodules developing around these [2] and pathogenicity is mainly due to these larvae. Prepatent period of $O$. columbianum is 41 days [3] which is longer than other gastrointestinal (GI) nematodes which in most of the cases is 2-3 weeks.

$O$. columbianum is found as mixed infection with other nematodes particularly Haemonchus

Copyright: The authors. This article is an open access article licensed under the terms of the Creative Commons Attributin License (http:// creative commons.org/licenses/by/2.0) which permits unrestricted use, distribution and reproduction in any medium, provided the work is properly cited. contortus and other helminths like Paramphistomum epiclitum which also shares intestinal habitat with it. Fasciola gigantica is also found in some regions in India. Clinical symptoms of these infections are overlapping making specific diagnosis difficult. Although, studies on immunodiagnosis of $O$. columbianum was reported previously by few worker employing ELISA and double immunodiffusion (DID) test [4-8]. Antigenic cross-reactivity of $O$. columbianum with. $H$. contortus was reported using DID and similar results employing ELISA [4]. Surface reactivity patterns among strongylid nematodes using immunoperoxidase assay revealed cross antigenicity of $O$. columbianum with $H$. contortus and Bunostomum trigonocephalum [9].

Hence, immunodiagnosis with a suitable noncross antigenic molecule may be a suitable alternative for specific diagnosis and timely treatment to prevent morbidity and mortality of infected animals. Cross antigenicity among mixed helminths infection has already been reported by Philipp and Rumjaneck [10]; Cuquerella et al. [11]; Molina et al. [12]. Keeping in view the pathogenic importance of $O$. columbianum in sheep and goat, the present study was conducted to 
identify non-cross antigenic polypeptide to be utilized for immunodiagnosis further.

\section{Materials and Methods}

\section{Ethical approval}

All the procedures have been carried out in accordance with the guidelines laid down by the Institutional/Animal Ethics Committee and in accordance with local laws and regulations.

\section{Collection of parasites}

Adult $O$. columbianum were collected from the intestines of infected sheep and goat having pimply gut (Figure-1) procured from the local abattoir at Bareilly (Uttar Pradesh) India. The parasites were washed several times in phosphate buffered saline (PBS - $\mathrm{pH}$ 7.2).

\section{Identification of $\boldsymbol{O}$. columbianum}

O. columbianum was identified as per their characteristic features $[13,14]$.

\section{Preparation of somatic antigen (SoAg)}

The adult worms were thoroughly homogenized using mortar and pestle in sufficient quantity of PBS $(\mathrm{pH} 7.2)$, then sonicated and further centrifuged at $12000 \mathrm{rpm}$ at $4^{\circ} \mathrm{C}$ for $25 \mathrm{~min}$. The supernatant was collected and filtered through $0.2 \mu \mathrm{m}$ (Sartorius) filter membrane. The filtrate was stored at $-70^{\circ} \mathrm{C}$ with PMSF.

\section{Protein estimation}

The protein concentration of the antigens was estimated as per Lowry's method [15].

Characterization of SoAg by sodium dodecyl sulfate-polyacrylamide gel electrophoresis (SDS-PAGE)

SDS-PAGE was performed to observe the polypeptide profile of the antigen of $O$. columbianum. Molecular weights of the polypeptides were determined with help of standard marker 11-170 KDa (Fermentas) as per the given method [16]. $40 \mu \mathrm{g}$ of protein of each antigen sample was mixed with $\times 1$ sample buffer solution in the ratio of $1: 1$. The antigens with sample buffer $(50 \mu 1)$ were charged into the wells of the gel. Molecular weight marker $(10 \mu \mathrm{l})$ was

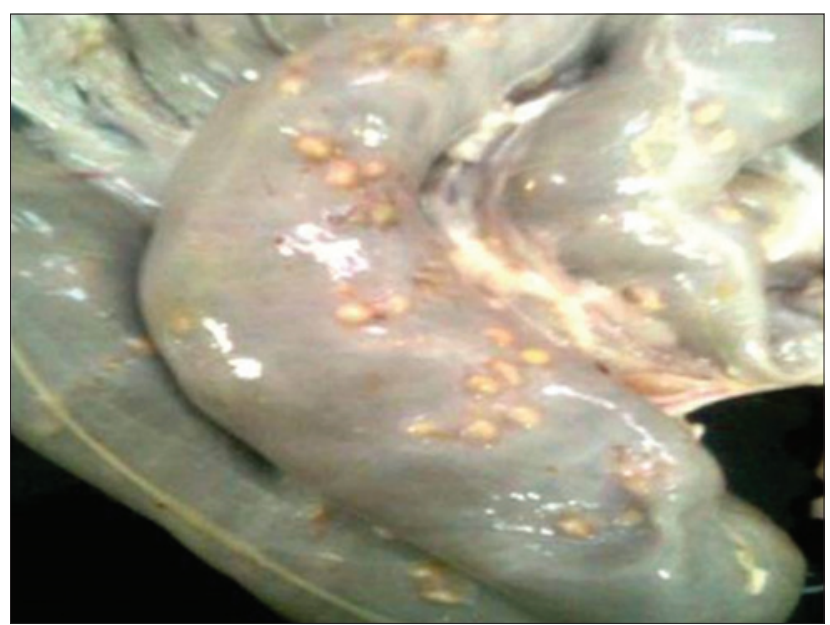

Figure-1: Pimply gut in colon of sheep. also loaded in the respective well. After the electrophoretic run, the gel was carefully removed from the glass plate and stained with coomassie blue R-250 for $30 \mathrm{~min}$ until stained properly. It was followed by destaining until the polypeptide bands were clearly visible.

\section{Hyperimmune sera (HIS)}

HIS was raised against SoAg of O. columbianum in PBS (pH 7.2) in New Zealand white rabbits as per the procedure with control [17]. Blood samples were collected from all the rabbits intracardially before immunization to obtain preimmunized normal rabbit sera and stored at $-20^{\circ} \mathrm{C}$. For raising HIS, stable water in oil emulsion of an equal volume of antigen Freuend's complete and Freuend's incomplete adjuvants were used as inoculums. Dose and route of administration of antigen and adjuvant were used as per standard protocol. Primary immunization (0 day) was done with subcutaneous injection with $450 \mu \mathrm{g} /$ animal. Further, booster doses were given at an interval of 12 days with increasing dose of $50 \mu \mathrm{g}$ protein from first to nine boosters by intramuscular injection. Finally, HIS were collected from immunized animals.

\section{Western blotting}

Western blotting was performed using $7 \mathrm{~min}$ i-blot (Invitrogen). SDS-PAGE gel was removed carefully from the glass plates and kept in triple distilled water for $10 \mathrm{~min}$. Polypeptides from the gel were transferred to nitrocellulose (NC) membrane $(0.2 \mu \mathrm{m})$. The membrane was kept in blocking buffer (PBS-M) for $2 \mathrm{~h}$ and washed with washing solution (PBS-T) 3 times with $5 \mathrm{~min}$ interval. The membrane was incubated with anti - serum $(1: 100)$ at $37^{\circ} \mathrm{C}$ for $2 \mathrm{~h}$ and washed 3 times with washing solution. Secondary antibody with 1:10000 dilution (Goat anti- Rabbit IgG HRP conjugate) was added to the membrane and incubated at room temperature for $1 \mathrm{~h}$ followed by final washing. 3, 3'-diaminobenzidine substrate was further added for developing bands of reacting polypeptides.

\section{Immunoaffinity purification of SoAg}

SoAg was purified using aminolink matrix (Pierce). The column containing matrix was equilibrated at room temperature and regenerated by washing with coupling buffer. The column was loaded with purified immunoglobulin (Ig) $(5 \mathrm{mg} / \mathrm{ml})$. IgG was separated from the HIS raised in rabbit against SoAg of O. columbianum. For purification of $\mathrm{IgG}$, protein $\mathrm{G}$ based IgG purification kit (Merck) was used and from $10 \mathrm{ml}$ HIS approximately $20 \mathrm{mg}$ IgG was obtained. $20 \mathrm{mg}$ purified Ig was loaded in the column and $200 \mu \mathrm{l}$ of aminolink reductant solution was added. It was incubated overnight at $4{ }^{\circ} \mathrm{C}$ with end to end shaking. Next day, the column was washed to remove excess uncoupled antibody. After complete washing, the antigen $(5 \mathrm{mg} / \mathrm{ml})$ was loaded to the column and incubated overnight at $4^{\circ} \mathrm{C}$ with end to end shaking. Excess uncoupled Ag was removed with washing buffer and finally the bound polypeptides were eluted 
utilizing elution buffer and fractions were collected. OD values of the fractions (Graph-1) were recorded, and the fractions of interest were pooled and concentrated followed by recording the OD values of pooled and concentrated fraction.

\section{SDS-PAGE of purified fractions}

SDS-PAGE of immunoaffinity purified fractions of the SoAg was performed as described above.

\section{Western blotting for cross antigenicity}

Western blot was performed with eluted purified fractions of SoAg with heterologous HIS as well as experimental sera (sheep and goat) raised against $H$. contortus, P. epiclitum, and F. gigantica and polypeptides showing reactivity to heterologous sera were identified for further identification of non-cross antigenic polypeptides in $O$. columbianum antigen. Experimental sera of sheep and goat against $H$. contortus, P. epiclitum, and F. gigantica were also utilized for western blotting.

\section{Results}

\section{Identification of parasite}

The worms showed cuticle forming mouth collar in form of truncated cone. The cervical groove was extending around ventral surface of the body and cuticle anterior to this groove inflated to form cephalic vesicle. Lateral alae originated immediately behind cervical groove, extending almost whole length of the body and cervical papillae pierced the anterior extremities of the lateral alae. The buccal capsule was shallow, external corona radiata consisting of 20-24 elements with internal corona radiata having two small elements to each element of external leaf crown. Oesophagus was club-shaped with oesophageal intestinal valve. Male bursa was well-developed with equal sized spicules not extending from bursal lobe. Vulva of a female was slightly prominent opening little anterior to anus. Vagina was very short, transverse, leading into kidney shaped ovijectors or pars ejectrix. The morphological features confirmed to the description of $O$. columbianum (Figure-2).

\section{Antigen preparation and protein concentration}

SoAg was prepared by standard protocol and the protein concentration was $3.32 \mathrm{mg} / \mathrm{ml}$ as per Lowry method.

\section{Antigen characterization of 0 . columbianum by SDS-PAGE}

Protein profile of SoAg of adult $O$. columbianum as determined by $12 \%$ SDS-PAGE revealed prominent protein bands of 11-130 kDa.

\section{Identification of immunodominant polypeptides in SoAg of $O$. columbianum by western blotting}

Protein bands fractionated by SDS-PAGE were subjected to western blotting using HIS raised against So Ag. Polypeptide bands of 17, 28, 30, 32, $35,38,50,68,100,130$, and $150 \mathrm{KDa}$ showed strong reactivity and were designated as immunodominant polypeptides.

\section{SDS-PAGE of immunoaffinity purified SoAg}

Protein profile of purified fractions of SoAg was determined by $12 \%$ SDS-PAGE. Sharp bands of 130 , 72 and $68 \mathrm{kDa}$ could be observed along with several faint bands of lower molecular weight (Figure-3).

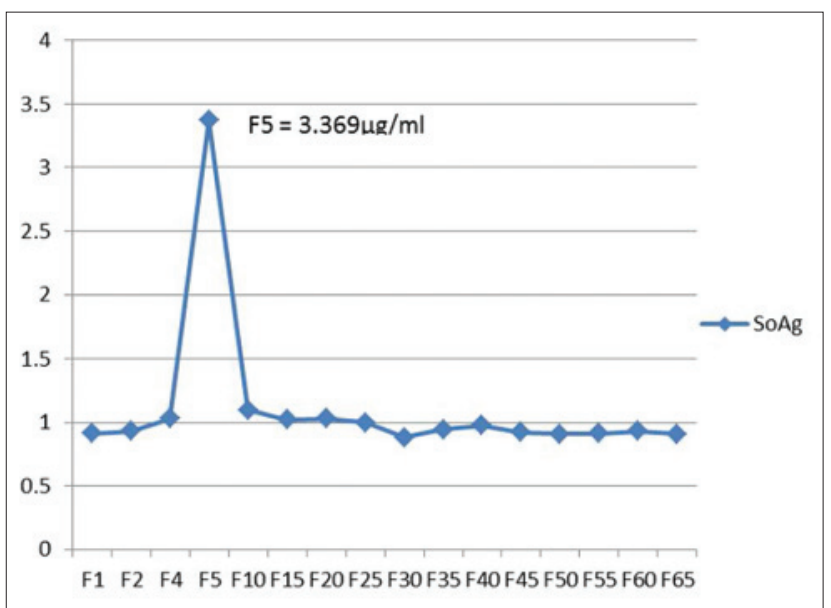

Graph-1: Elution pattern of bound fractions of purified somatic antigen of Oesophagostomum columbianum.

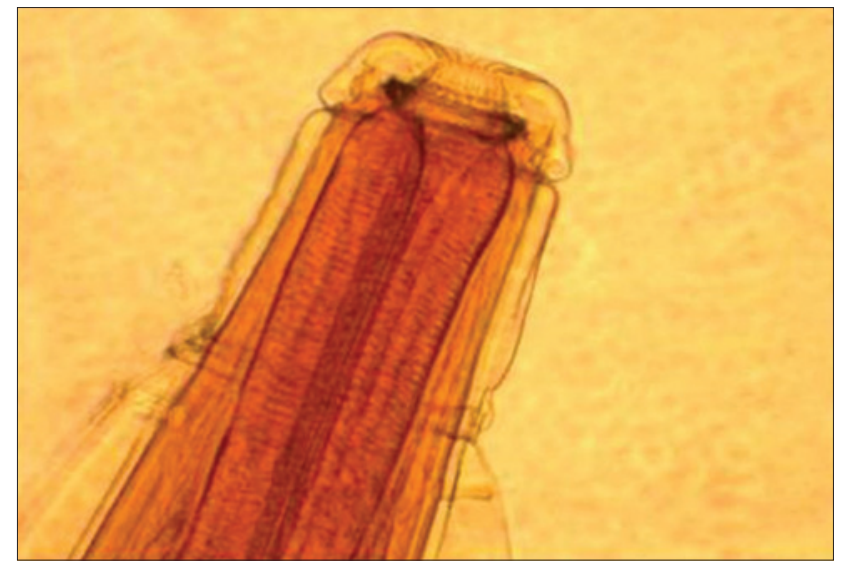

Figure-2: Anterior end of Oesophagostomum columbianum (cervical papillae and leaf crown).

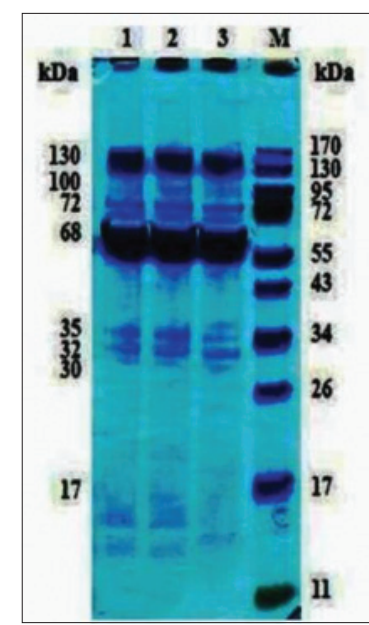

Figure-3: Sodium dodecyl sulfate-polyacrylamide gel electrophoresis of immunoaffinity purified somatic antigen of Oesophagostomum columbianum, Lane: 1, 2 and 3: Immuno affinity purified fraction, Lane: M: Moecular weight marker. 
Western blot with immunoaffinity purified SoAg of O. columbianum with homologous HIS

Western blot of purified SoAg of O. columbianum with homologous HIS showed reaction with all the protein bands of $17,28,30,32,35,38,50,68,100$, 130, 150 and $170 \mathrm{kDa}$ (Figure-4).

Immunoaffinity purified SoAg of 0 . columbianum and $\mathrm{HIS}$ against $\boldsymbol{H}$. contortus SoAg

HIS against $H$. contortus reacted to 130,50 , and $28 \mathrm{KDa}$ polypeptide present in the immunoaffinity purified SoAg of O. columbianum (Figure-5).

Immunoaffinity purified SoAg of 0 . columbianum and experimental sera of sheep against $\boldsymbol{H}$. contortus

The experimental serum of sheep against $H$. contortus reacted only to 130 and $50 \mathrm{KDa}$ polypeptide of purified SoAg of O. columbianum (Figure-6).

Immunoaffinity purified SoAg of 0 . columbianum and HIS against $\boldsymbol{P}$. epiclitum

HIS against $P$. epiclitum reacted to polypeptides of 130, 68 and $50 \mathrm{KDa}$ of purified SoAg of O. columbianum (Figure-7).

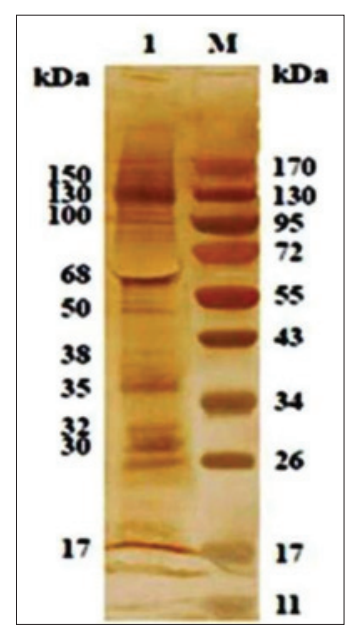

Figure-4: Western blotting of immunoaffinity purified somatic antigen of Oesophagostomum columbianum with homologous hyperimmune serum.

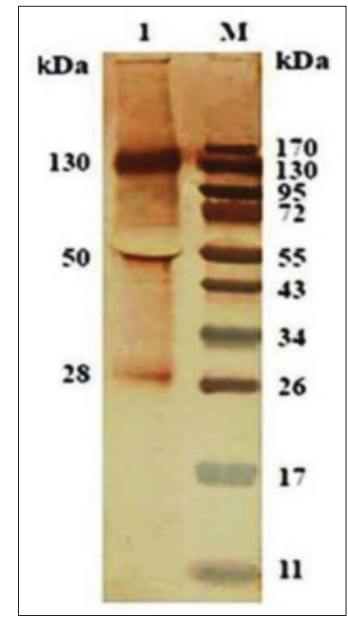

Figure-5: Cross-antigenicity of immunoaffinity purified somatic antigen of Oesophagostomum columbianum with anti-Haemonchus contortus antibody (hyperimmune serum) in western blotting.
Immunoaffinity purified SoAg of 0 . columbianum and experimental sera of goat against $\boldsymbol{P}$. epiclitum

The experimental serum of goat against $P$. epiclitum reacted to 130,68 , and $50 \mathrm{KDa}$ polypeptides in the purified SoAg of O. columbianum (Figure-8).

Immunoaffinity purified SoAg of 0 . columbianum and experimental sera of sheep against $\boldsymbol{F}$. gigantica

Experimental serum of sheep against $F$. gigantica reacted with 130, 68 and 50 KDa polypeptides of purified SoAg of $O$. columbianum (Figure-9).

The results of cross antigenicity of $O$. columbianum have been summarized in Table-1.

\section{Discussion}

Non-cross antigenic immunodominant polypeptides in SoAg of O. columbianum were identified by western blotting. In affinity purified fraction, major prominent protein bands were $68,72,100$, and $130 \mathrm{kDa}$, whereas low molecular weight polypeptides formed feeble bands. Semi dry method for western

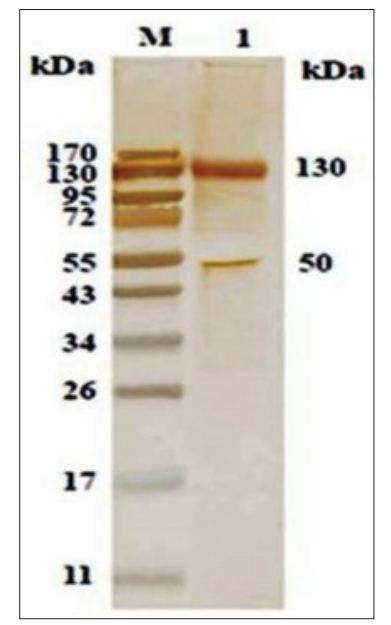

Figure-6: Cross antigenicity of immunoaffinity purified somatic antigen of Oesophagostomum columbianum with experimental serum of sheep infected with Haemonchus contortus in western blotting.

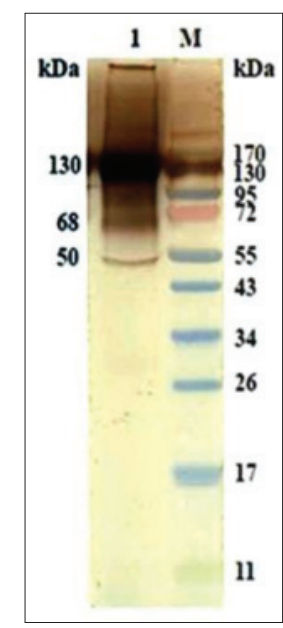

Figure-7: Cross antigenicity of immunoaffinity purified somatic antigen of Oesophagostomum columbianum with anti-Paramphistomum epiclitum antibody (hyperimmune serum) in western blotting. 


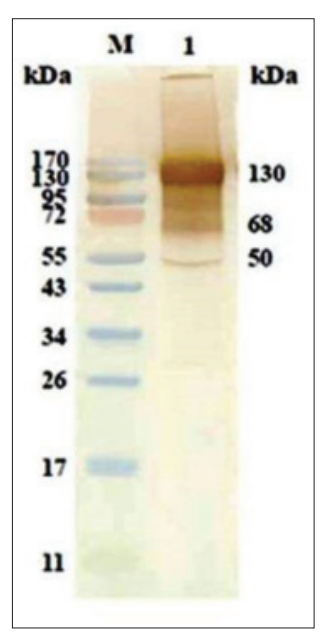

Figure-8: Cross-antigenicity of immunoaffinity purified somatic antigen of Oesophagostomum columbianum with experimental serum of Paramphistomum epiclitum in western blotting.

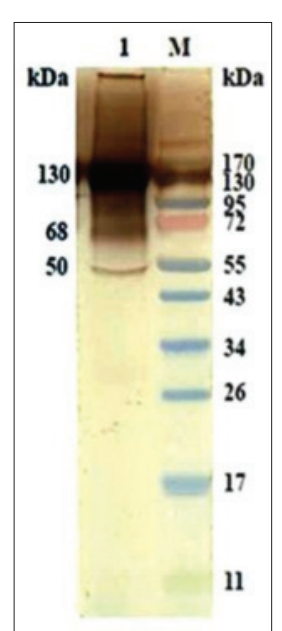

Figure-9: Cross-antigenicity immunoaffinity purified somatic antigen of Oesophagostomum columbianum with experimental serum of Fasciola gigantica in western blotting.

Table-1: Cross-antigenicity of different helminthes with immunoaffinity purified SoAg of $O$. columbianum.

\begin{tabular}{lc}
\hline Heterologous serum & $\begin{array}{c}\text { Cross antigenic } \\
\text { polypeptide (kDa) }\end{array}$ \\
\hline P. epiclitum (HIS) & $130,68,50$ \\
P. epiclitum & $130,68,50$ \\
(experimental serum in goat) & $130,50,28$ \\
H. contortus (HIS) & 130,50 \\
H. contortus & $130,68,50$ \\
(experimental serum in sheep) & \\
F. gigantica & (experimental serum in goat)
\end{tabular}

$P$. epiclitum = Paramphistomum epiclitum, F. gigantica $=$ Fasciola gigantica, $\mathrm{H}$. contortus=Haemonchus contortus, O. columbianum=Oesophagostomum columbianum, SoAg=Somatic Antigen, HIS=Hyperimmune serum

blotting is routinely used to transfer polypeptides from SDS-PAGE gel, but incomplete transfer of polypeptides was frequently encountered. To overcome the problem, completely dry method i-blot (Invitrogen) was utilized. This method had several advantages and most important was time interval ( $7 \mathrm{~min}$ ) as compared to semi-dry method taking few hours for complete transfer of polypeptides. Therefore, for cross antigenicity studies completely dry method was utilized with the objective that all the polypeptides transferred were available for reaction to antibodies. It was observed that in western blotting polypeptides of lower as well as higher molecular weight were transferred to $\mathrm{NC}$ membrane and all the antigenic polypeptides on the membrane reacted to antibodies. During this study, the polypeptides of higher molecular weight $(68,72$, 100,130 , and $150 \mathrm{kDa}$ ) and lower molecular weight $(17,30,32,35$, and $38 \mathrm{kDa})$ were observed in the SDS-PAGE of affinity purified fraction of $O$. columbianum. During the study, it was found that polypeptides of lower molecular weight $(17,30,32,35$, and $38 \mathrm{kDa}$ ) reacted to HIS against $O$. columbianum (homologous sera) but not to heterologous sera in western blotting. Besides, few polypeptides of higher molecular weight (100 and $150 \mathrm{kDa}$ ) also did not react to heterologous sera in western blotting raised against $H$. contortus, $P$. epiclitum and F. gigantica. It was inferred that there were several polypeptides both of lower as well as higher molecular weight in the SoAg of $O$. columbianum which were not shared with other helminths. In a similar study [18] found that out of 46 goats, five goats, which were negative for $O$. columbianum, showed false positive reaction in the assay since the goats although negative for $O$. columbianum had heavy worm burden of $H$. contortus (170-265) and Trichuris ovis (143-239). The authors suspected possibility of cross antigenicity with $H$. contortus and $T$. ovis due to reaction between HIS against ESAg-Oc and coproantigen (cAg) of H. contortus and T. ovis. The observation of these workers was found true during the present study because in western blotting, $50 \mathrm{kDa}$ polypeptide of $O$. columbianum reacted with the HIS and experimental heterologous sera against $H$. contortus, $P$. epiclitum, and $F$. gigantica which confirmed cross antigenicity of $O$. columbianum with these helminths. Jas et al. [18] did not perform cross antigenicity studies. 39 and $50 \mathrm{kDa}$ polypeptides were found immunodominant in the ES product of $O$. columbianum in western blotting with homologous HIS [18] however, 29, 38 and $49 \mathrm{kDa}$ were found immunodominant by Arunkumar [19]. During our study, it was found that $50 \mathrm{kDa}$ polypeptide of SoAg of $O$. columbianum was cross antigenic with $H$. contortus, $P$. epiclitum, and $F$. gigantica. This was actually the reason that goats negative for $O$. columbianum were giving false positive reaction in ELISA [18].

During this study, it was found that cross antigenicity of purified fraction of $O$. columbianum with $H$. contortus, $P$. epiclitum, and $F$. gigantica were mainly among high molecular weight polypeptides. Results of western blotting with heterologous hyperimmune sera and experimental sera of sheep and goat revealed that not only polypeptides of low molecular 
weight $(17,30,32,35$, and $38 \mathrm{kDa})$ but also of high molecular weight (100 and $150 \mathrm{kDa}$ ) were non-cross antigenic to other helminths.

Sharing of polypeptides among GI nematodes has already been described by Siefker and Rickard [20], who recorded sharing of carbohydrate epitopes in an intestinal protein of bovine GI nematodes. In western blotting, these workers recognized sharing of high molecular weight protein bands ranging between 111 -298 KDa of Haemonchus placei of cattle with several nematodes such as Ostertagia ostertagi, Cooperia punctata, $H$. contortus, and Oesopohagostomum radiatum. These polypeptides were conserved in these species. In $O$. radiatum the polypeptides were semi-conserved. They also confirmed their finding with immunohistochemical studies verifying the intestinal location of the epitopes of the antigens. Cross antigenicity of $O$. columbianum during the present study was found with common trematodes $F$. gigantica and $P$. epiclitum in western blotting. Cross antigenicity of SoAg of $H$. contortus with $O$. columbianum and $F$. gigantica has already been reported by Prasad et al. [21] but not with P. epiclitum. Non-cross antigenic polypeptides in the SoAg were mostly low molecular weight polypeptides. Results indicate that for immunodiagnosis of $O$. columbianum infection, low molecular weight polypeptides $17,30,32,35$, and $38 \mathrm{kDa}$ may be exploited. Jas et al. [18] found only $39 \mathrm{kDa}$ polypeptide of ES antigen reacting to homologous hyperimmune sera against O.columbianum. Other polypeptides like 32 and $35 \mathrm{kDa}$ probably were not available for recognition by antibodies due to failure of transfer during western blotting. However, during the present study, high molecular weight polypeptides of 100 and $150 \mathrm{kDa}$ were also found noncross antigenic.

\section{Conclusion}

Immunodiagnosis of O.columbianum is rather difficult proposition since not much work has been done due to its importance mainly in tropical and subtropical countries The infection causes pimply gut in sheep and goat and causes severe diarrhea. Low molecular weight polypeptides of $17,30,32$, 35 , and $38 \mathrm{kDa}$ were found non-cross antigenic with $H$. contortus, P. epiclitum, and F. gigantica in western blotting utilizing heterologous HIS, as well as experimental sera. Among high molecular weight polypeptides, 100 and $150 \mathrm{kDa}$ were found non-cross antigenic. Further, work is therefore needed to explore diagnostic potential of these polypeptides with extensive studies on cross antigenicity to develop a specific immunodiagnostic test for $O$. columbianum infection in sheep and goat.

\section{Authors' Contributions}

SD: Preparation of antigen, SDS PAGE, purification of antigen and cross antigenicity studies. AP: Western blotting, cross antigenicity studies and preparation of manuscript. AN: Immunoaffinity purification raising of hyperimmune serum in rabbit and preparation of manuscript. VKS: Preparation of manuscript and data analysis.

\section{Acknowledgments}

The authors are thankful to Director, IVRI, Izatnagar and Project Coordinator, Network Pogramme on G.I. Parasitism, Indian Council of Agricultural Research, New Delhi for providing necessary facilities for the work.

\section{Competing Interests}

The authors declare that they have no competing interests.

\section{References}

1. Mohanta, U.K., Anisuzzaman, F.T., Das. P.M., Majumder, S. and Mondal, M.M.H. (2007) Prevalence, population dynamics and pathological effects of intestinal helminthes in black Bengal goats. Bangladesh J. Vet. Med., 5(1-2): 63-69.

2. Dash, K.M. (1973) The life cycle of Oesophagostomum columbianum (Curtice, 1890) in sheep. Int. J. Parasitol., 3: 843-851.

3. Soulsby, E.J.L. (1982) Helminths Arthropods and Protozoa of Domesticated Animals. $7^{\text {th }}$ ed. The English Language Book Society and Bailliere, Tindall, London.

4. Lone, B.A., Chisti, M.Z., Ahmed, F. and Hidayatullah, T. (2011) Serodiagnosis of Oesophagostomum columbianum infection in goats using indirect ELISA. Vet. World., 4(11): 503-506.

5. Lone, B.A., Chisti, M.Z., Ahmed, F., Tak, H. and Hassan, J. (2012) Immunodiagnosis of Haemonchus contortus infection in sheep by indirect enzyme linked immunosorbent assay (ELISA). Iran. J. Vet. Res., 13 (1): 49-53.

6. Arunkumar, S. and Sangaran, A. (2010) Evaluation of antigenic cross-reactivity amongst strongy lid nematodes using enzyme linked immunosorbent assay. Indian J. Small. Rumin., 16(1): 131-133.

7. Jas, R., Ghosh, J.D. and Das, K. (2010) Diagnosis of Oesophagostomum columbianum infection in goat by indirect enzyme linked immunosorbent assay. Helminthologia, 47(2): 83-87.

8. Arunkumar, S. and Sharma, R.L. (2010) Assessment of antigenic cross reactivity amongst strongylid nematodes using ouchterlony double immunodiffusion test. Indian J. Field. Vet., 5(4): 47.

9. Arunkumar, S. and Sharma, R.L. (2010) Surface reactivity patterns amongst strogylid nematodes using immunoperoxidase assay. Indian J. Field. Vet., 6(1): 36.

10. Philipp, M. and Rumjaneck, F.D. (1984) Antigenic and dynamic properties of helminth surface structures. Mol. Biochem. Parasitol., 10: 245-268.

11. Cuquerella, M., Munoz, M.T.G., Carrea, L., Fuente, C.D.L. and Alunda, J.M. (1994) Cross antigenicity among ovine Trichostrongyloidea. Prelimanary report. Vet. Parasitol., 53: 243-251.

12. Molina, J.M., Ruiz, A., Rodriguez-Ponce, E., Guterrez, A.C., Gonzalez, J. and Hermandez, S. (1999) Cross reactive antigens of Haemonchus Contortus adult worms in Teladorsagia circumcincta infected goats. Vet. Res., 30: 393-399.

13. Yamaguti, S. (1961) Systema Helminthum. Inter Science Publishers Ltd., London. p433-434.

14. Levine, N.D. (1980) Nematode Parasites of Domestic Animals and of Man. Burgers Publishing Company Minneapolis, Minnesota.

15. Lowry, O.H., Rosebrough, N.J., Farr, A.L. and Randall, R.J. (1951) Protein measurement with folin phenol reagent. $J$. 
Bio. Chem., 193: 265-268.

16. Laemmli, U.K. (1970) Cleavage of structural proteins during the assembly of bacteriophage $\mathrm{T}_{4}$. Nature, 227: 680-685.

17. Hundson, L. and Hay, F.C. (1989) Practical Immunology. $3^{\text {rd }}$ ed. Blackwell Scientific Publications, Oxford, London. p507.

18. Jas, R., Ghosh, J.D. and Das, K. (2010) Polyclonal antibody based coproantigen detection immunoassay for diagnosis of Oesophagostomum columbianum infection in goats. Vet. Parasitol., 170: 262-267.

19. Arunkumar, S. (2012) Antigenic characterization of excretory/secretory antigen of Oesophagostomum columbianum using western blotting. CIB Tech J. Biotechnol., 1(2 \& 3): 28-31.

20. Siefker, C. and Rickard, L.G. (1998) Demonstration of shared carbohydrate epitopes in intestinal proteins of some bovine gastro intestinal nematodes. J. Parasitol., 84(3): 524-552.

21. Prasad, A., Nasir, A. and Singh, N. (2007) Dot-ELISA for detection of prelinical Haemonchus contortus infection in sheep with adult somatic antigen and immunoaffinity purified fraction. J. Parasit. Dis., 31(1): 22-28.

$* * * * * * * *$ 